\title{
Oral iloprost as a treatment for Raynaud's syndrome: a double blind multicentre placebo controlled study
}

J J F Belch, H A Capell, E D Cooke, J D T Kirby, C S Lau, R Madhok, E Murphy, M Steinberg

\begin{abstract}
Objective-To compare the efficacy, tolerance and safety of 50-150 $\mu \mathrm{g}$ orally administered iloprost given twice a day versus placebo in patients with Raynaud's syndrome.

Methods-The study was multicentre $(n=3)$, double blind and placebo controlled. Sixty three patients who had eight or more vasospastic attacks per week were enrolled. After a one week run-in period, all patients received either iloprost or placebo treatment to a maximum tolerated dose of $150 \mu \mathrm{g}$ twice a day for 10 days. Diary cards assessed the duration and severity of the vasospastic attacks. Side effects were monitored by direct questioning. A global assessment of treatment efficacy was made by the patient at the end of treatment and two weeks later.
\end{abstract}

Results-Patient opinion tended to favour iloprost at the end of the $\mathbf{1 0}$ day treatment phase $(p=0.09)$ and this was significant at day 24 (the follow up visit) $(p=0 \cdot 011)$. Although the duration and severity of attacks tended to decrease in the iloprost treated group, these results tended not to reach statistical significance (for severity $p=0.06$ at end of treatment, $p=0.09$ on day 24).

University Department of Medicine, Ninewells Hospital and Medical School, Dundee DD1 9SY, United Kingdom J J F Belch

C S Lau

Centre for Rheumatic Diseases,

Royal Infirmary,

Glasgow G4 OSF,

United Kingdom

H A Capell

R Madhok

E Murphy

Thermographic and

Blood Flow Unit,

Department of Medical

Electronics,

St Bartholomews

Hospital,

London EC1A 7BE,

United Kingdom

E D Cooke

M Steinberg

Department of

Dermatology

J D T Kirby

Correspondence to:

Dr Belch.

Accepted for publication 12 October 1994 but this route of administration is inconvenient. This study evaluated the use of iloprost administered orally to patients mented improvement was significantly improved by iloprost. Diary card analysis showed a trend in favour of iloprost, but these results did not reach statistical significance.

(Ann Rheum Dis 1995; 54: 197-200)

Raynaud's phenomenon is characterised by episodic digital ischaemia provoked by cold and emotion. ${ }^{1}$ It may be idiopathic, Raynaud's disease, or associated with a wide variety of other conditions, particularly the connective tissue disorders. ${ }^{2}$ This secondary form, Raynaud's syndrome (RS), is a debilitating condition which can seriously interfere with the sufferer's quality of life. Progression to digital ulceration and even gangrene can occur, which may lead to loss of the digits.

Treatment of RS can be difficult and no completely satisfactory approach has been developed. Vasodilatory therapy has been superseded by the use of calcium channel antagonists such as nifedipine; ${ }^{3}$ although these can be very useful they may have troublesome side effects and are ineffective in severely affected patients. ${ }^{4}$ Several studies have shown that prostacyclin $\left(\mathrm{PGI}_{2}\right)$, a potent vasodilator antiplatelet agent, can reduce the severity and frequency of Raynaud's attacks and that these effects are prolonged for several weeks after the infusion. ${ }^{5} \mathrm{PGI}_{2}$ is chemically unstable, however, and has a half-life of 2-3 minutes, and this has limited its clinical use. Iloprost (Schering Chemicals Ltd) is a chemically stable $\mathrm{PGI}_{2}$ analogue and thus has advantages because of its relative ease of use. It has been shown to be effective in the treatment of RS when given intravenously, ${ }^{78}$ and is equipotent with nifedipine. ${ }^{4}$ It appears to be effective against vasospasm even when given in low dose, ${ }^{8}$ and may produce significant healing of digital ulcers. ${ }^{9}$ Despite this, it still remains a second choice treatment because of the need for intravenous administration.

A slow release orally active preparation of iloprost is now available and the aim of the study was to evaluate the safety, tolerance, and efficacy of orally administered iloprost in patients with RS secondary to systemic sclerosis (SSc). The study was multicentre, placebo controlled and double blind. with Raynaud's syndrome. Patient docu-

\section{Patients and methods}

Three centres participated in the study (Dundee, Glasgow, and London). All centres followed identical core protocols so that a meta-analysis of the clinical and safety data could be made. Sixty three patients fulfilled enrolment criteria of the study. All had RS secondary to SSc. RS was defined as intermittent attacks of blanching and cyanosis of the digits precipitated by exposure to cold or emotion, and patients were required to have at least eight attacks per week to be included in the study. SSc was diagnosed by the presence of either proximal scleroderma or two of the following: sclerodactyly, digital pitting or loss of pad, bilateral basilar pulmonary fibrosis, and abnormal nailfold capillaries assessed by capillaroscopy. The American Rheumatologists' 
Association criteria were not used for enrolment into this study as patients fulfilling these have severe disease, often with no vasodilator reserve, and were felt to be less likely to respond to the effects of iloprost. All female patients $(n=58)$ of child bearing potential were required to have a negative pregnancy test before inclusion and to be taking adequate contraceptive measures. All patients gave informed written consent and ethical permission for the study was obtained from the regional ethics committees of all three centres.

After a washout period of two weeks, during which no medication for relief of Raynaud's symptomatology was taken, patients entered the study. Diary cards were issued and the patients asked to record, on a daily basis, the duration of their Raynaud's attacks in minutes, whether the attack was mild, moderate or severe, and whether it was painful or not. These were completed for a seven day run-in period to ensure that at least eight attacks per week were documented. If an error in completion was detected, this seven day run-in period was repeated. Diary card documentation continued throughout the study period to day 24 .

Medication was issued after this run-in period and continued for 10 days. The 10 day limit on treatment duration is a standard limitation imposed by the regulatory authorities on introduction of a novel compound into a patient population. The patients were formally assessed at the start of the 10 day treatment period, at day 3, day 10 , and two weeks after the cessation of treatment at day 24. All were inpatients for the first three days and two nights of the study so that side effects could be monitored and blood pressure and pulse rate measured. Blood was taken for routine haematology and biochemistry (urine and electrolytes, liver function tests) on day 0 and day 10. A pregnancy test was repeated in females of child bearing potential on day 10 and also on this day the patient's opinion as to treatment efficacy was sought. They were asked if the condition had improved, not changed, or worsened compared with before treatment. The study was conducted between January and April and all patients were asked to continue with their usual lifestyle including, for example, use of handwarmers or electric gloves.

STUDY MEDICATION AND ADMINISTRATION Iloprost was supplied by Schering Chemicals Ltd as capsules of $50 \mu \mathrm{g}$. Matching placebo capsules were also provided. The study medi-

Table 1 Comparability of groups

\begin{tabular}{lll}
\hline & Placebo & Iloprost \\
\hline No of patients & 31 & 32 \\
Sex: Male/Female & $3 / 28$ & $2 / 30$ \\
Age (yr) & $49(44-54)$ & $52(47-57)$ \\
Total duration of attacks (min) & $809(549-1070)$ & $918(380-1456)$ \\
Total number of attacks/week & $22(17-27)$ & $24(18-30)$ \\
Number of painful attacks as a proportion of all attacks & $0 \cdot 5(0 \cdot 3-0 \cdot 6)$ & $0 \cdot 5(0 \cdot 3-0 \cdot 6)$ \\
Average severity of attacks on scale $0-3$ & $1 \cdot 9(1 \cdot 6-2 \cdot 1)$ & $1 \cdot 9(1 \cdot 7-2 \cdot 2)$ \\
\hline
\end{tabular}

Values are mean (range) except for median age (range). cation was administered twice a day either at 08:00 and 14:00, or at 14:00 and 20:00. On the first day of treatment patients took one capsule twice, on the second day two capsules twice, and on the third day three capsules twice, corresponding to 50-150 $\mu \mathrm{g}$ twice a day or placebo. The greatest tolerated dose was used for the remaining seven days of treatment. Patients were allocated to receive either iloprost or placebo according to separate computer generated randomisation lists for all three centres.

\section{STATISTICAL ANALYSES}

The data were analysed using either the MannWhitney $\mathrm{U}$ test or the independent $t$ test, depending on the distribution of the data for continuous variables. The $\chi^{2}$ test was used for comparing categorical data between treatment groups. For analysis of the duration of Raynaud's attacks a percentage change from baseline was used. $\mathrm{p}<0.05$ was considered statistically significant.

\section{Results}

Table 1 shows that the two groups of patients were comparable in terms of age, gender, and Raynaud's attacks. Additionally, there were six smokers in the placebo group and nine smokers who received iloprost. Table 2 shows drug therapy for RS before the washout period; all medications were stopped before this two week period. Data from all 63 patients were available for the analysis of tolerance and safety data. Fifty three patients were included in the analysis of the efficacy of the drug; the 10 patients (seven placebo, three iloprost) excluded from this analysis failed to complete their diary cards correctly during the treatment phase of the study. Four patients (one placebo, three iloprost) withdrawn during the study had all their available data considered in the analyses; the three iloprost patients were withdrawn because of side effects and the placebo patient because of intercurrent illness.

\section{GLOBAL OPINION}

The patients' opinions of treatment are shown in table 2 and in the figure. In the placebo group almost $60 \%$ of the patients felt that the symptoms were unchanged or worse after two weeks follow up (day 24). In contrast, more than $60 \%$ of the patients who received iloprost felt their condition was improved. The difference between the treatments approached statistical significance $(p=0.09)$ at the end of treatment (day 10) and was statistically

Table 2 Raynaud's syndrome medication taken in the 14 days before enrolment into the washout phase

\begin{tabular}{lcc}
\hline & Placebo & Iloprost \\
\hline Nifedipine & 8 & 4 \\
Diltiazem & 2 & - \\
Amlodipine & 1 & - \\
Oxypentifyllin & - & 1 \\
Essential fatty acids & 2 & 10 \\
Total & 13 & \\
\hline
\end{tabular}




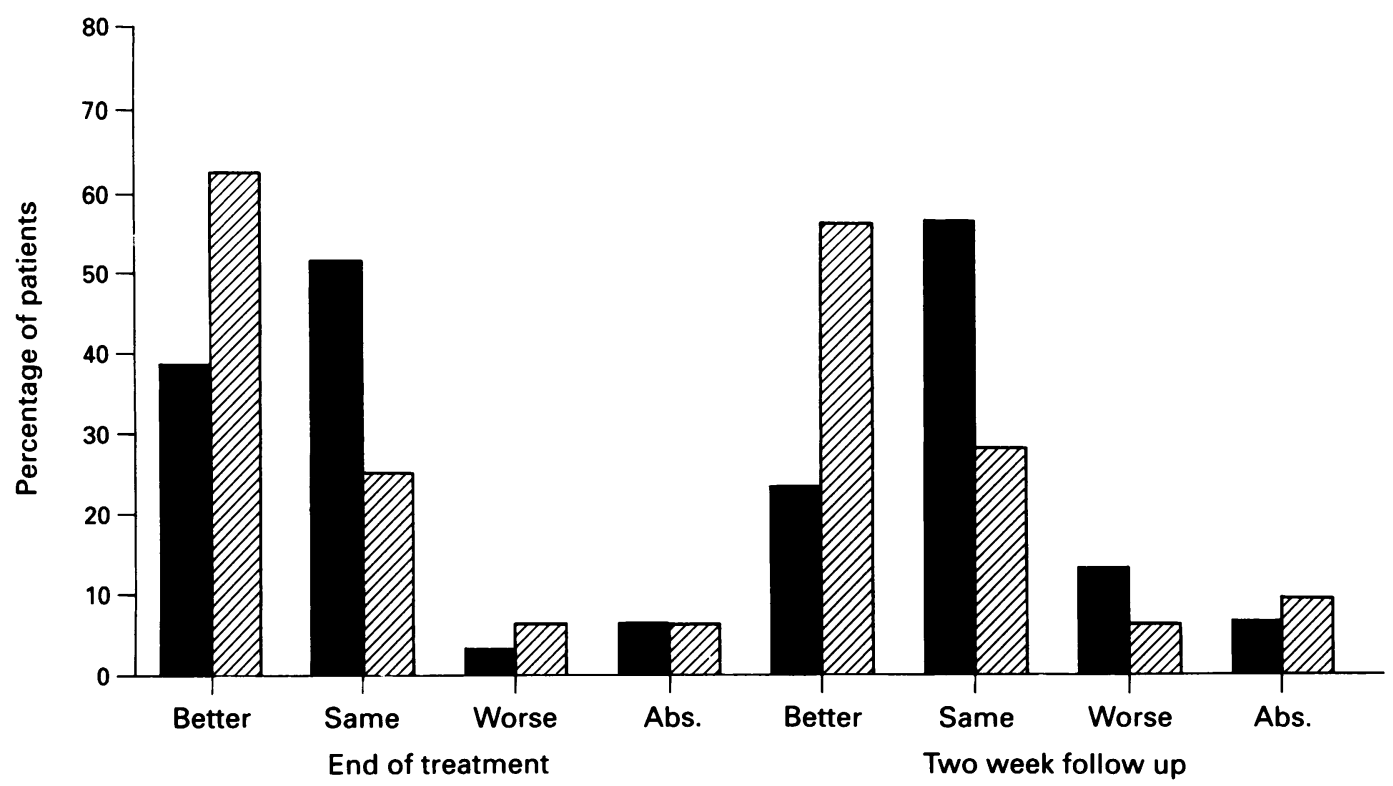

Patients' opinion of treatment with placebo (ם) or iloprost $(\square)$. Abs $=$ Absent. Difference between treatments almost significant $(p=0.09)$ at the end of treatment and significant $(p=0.011)$ at follow $u p$.

significant at the follow up visit (day 24) $(p=0.011)$.

\section{DURATION AND SEVERTYY OF RAYNAUD'S ATTACKS}

Table 3 shows the percentage change from baseline in the total duration of Raynaud's attacks per week. A greater reduction in mean total duration of attacks was seen in the iloprost group compared with placebo, but the difference did not reach statistical significance.

The severity of Raynaud's attacks was assessed by calculating the percentage change in the proportion of painful attacks and the change in average severity of the attacks using the scale: $0=$ none; $1=$ mild; $2=$ moderate; $3=$ severe (table 4). Iloprost treatment tended to cause a greater decrease in the number of painful attacks and a greater decrease in the severity of the attacks than placebo. At the end of treatment (day 10) the difference in

Table 3 Total duration of Raynaud's attacks: change from one week run-in baseline used to compare the duration of attacks per week

\begin{tabular}{lll}
\hline & \multicolumn{2}{l}{ Change in duration (\%) } \\
\cline { 2 - 3 } & Placebo & Iloprost \\
\hline $\begin{array}{l}\text { End of treatment } \\
\text { (week ending on day 10) }\end{array}$ & $-24(-43$ to -4$)$ & $-40(-57$ to -23$)$ \\
$\begin{array}{l}\text { Follow up } \\
\quad \text { During 1st week after treatment } \\
\text { During 2nd week after treatment }\end{array}$ & $-33(-50$ to -16$)$ & $-11(-45$ to -23$)$ \\
\hline
\end{tabular}

Values are mean ( $95 \%$ confidence interval). Negative sign indicates decrease from baseline. average severity of attacks between the groups just failed to reach statistical significance $(p=0 \cdot 06)$; it again approached significance at the end of the follow up period (day 24) $(p=0.09)$.

\section{ADVERSE EVENTS}

There were no serious adverse reactions or events as defined by the protocol. However, three patients in the iloprost group suffered adverse events that caused their withdrawal from the treatment phase of the study (one had severe headache, one had headache with nausea, and one had headache with flushing). During the three day inpatient phase a large number of minor adverse reactions were reported, the most common being headache, flushing, and nausea. Thirty one of 32 patients $(97 \%)$ receiving iloprost and 19 of 31 of placebo patients $(61 \%)$ reported one or more events during the treatment period $(\mathrm{p}<0.002)$ : $50 \%$ were classified as mild, $32 \%$ as moderate, and $18 \%$ severe. This high instance of side effects obliged the majority of patients to decrease their dose. The largest dose of three capsules twice daily was taken by $41 \%$ of the iloprost patients; $18 \%$ took the medium dose of two capsules twice daily and $41 \%$ chose to return to the smallest dose, of one capsule twice daily. At the selected doses all patients, except the three documented above, were able to continue with the medication.

Table 4 Pain and severity of Raynaud's attacks: change from baseline used to compare the pain and severity of attacks per week

\begin{tabular}{llllll}
\hline & \multicolumn{1}{l}{ Painful attacks } & & \multicolumn{1}{l}{ Severity } \\
\cline { 2 - 3 } & Placebo & Iloprost & & Placebo & Iloprost \\
\hline $\begin{array}{l}\text { End of treatment } \\
\quad \text { (week ending on day 10) }\end{array}$ & $-23(-50$ to 4$)$ & $1(-25$ to 27$)$ & & $-1(-7$ to 6$)$ & $-6(-12$ to -1$)$ \\
$\begin{array}{l}\text { Follow up } \\
\quad \text { During 1st week after treatment }\end{array}$ & $12(-75$ to 98$)$ & $-9(-30$ to 11$)$ & & $-1(-11$ to 9$)$ & $-5(-13$ to 3$)$ \\
During 2nd week after treatment & $-7(-60$ to 5$)$ & $-27(-53$ to -1$)$ & & $0(-9$ to 9$)$ & $-9(-18$ to -0$)$ \\
\hline
\end{tabular}

Values are mean ( $95 \%$ confidence interval). Negative sign indicates decrease from baseline. 


\section{Discussion}

The pathophysiology of Raynaud's phenomenon is not yet completely understood but is known to include contributions from abnormal neurogenic, ${ }^{10}$ blood and blood vessel wall, ${ }^{11}$ and immunological and inflammatory responses. ${ }^{12} 13$ The discovery that $\mathrm{PGI}_{2}$, produced by the vascular endothelium, is a potent vasodilator and inhibitor of platelet aggregation led to interest in the role of prostanoids in the treatment of RS. Such theoretical considerations were later supported by the finding of the anti-white ${ }^{14}$ and anti-red ${ }^{15}$ blood cell and fibrinolytic activities ${ }^{16}$ of prostacylin-all effects which might be expected to be useful in RS. Intravenous prostaglandins do appear to produce some benefit, ${ }^{6}$ but they can be difficult to use and are understandably less popular with patients than oral medications. Human volunteer studies with a new formulation of iloprost suitable for oral testing showed that, with the slow release preparation, $80 \%$ of the active substance was absorbed in the first hour, with a time to maximum plasma concentrations between 60 and 90 minutes. After a dose of $150 \mu \mathrm{g}$, therapeutic plasma concentrations were obtained for a period of 3.3 (SD 1.0) hours.

The present study was undertaken to investigate the efficacy, safety, and tolerance of the drug in RS patients. Although during treatment the reduction in duration of Raynaud's attacks was greater in the iloprost group, there were no statistically significant effects. The diary card data demonstrated a high placebo response of $25-30 \%$ for this variable. Furthermore, the wide $95 \%$ confidence intervals seen in table 3 indicated a high variability of the data-a feature often seen with the duration of attacks in RS. This would have necessitated a change of $60 \%$ from baseline in the iloprost group to achieve statistical significance. Conversely, the diary card data demonstrated a near significant result for iloprost for the severity of Raynaud's attacks, with these data showing less variability. This result is supported by the data obtained from the patients' opinions of improvement: the proportion of patients judging their symptoms to be improved was greater in the iloprost group. This difference approached significance at the end of treatment and was statistically significant at the two week follow up visit when the placebo effect in the control group had become attenuated.

Side effects were as expected and were related to the pharmacological properties of iloprost. ${ }^{17}$ Despite the number of reported side effects, only three patients felt them to be intolerable and withdrew from the treatment phase. The other patients reduced their drug dosage until the side effects were minimised. Forty percent of patients tolerated the maximum dose, whereas $60 \%$ did not. An increase in the maximum dose cannot therefore be suggested in an effort to achieve a greater clinical response. The titration of the dose over three days to a maximum of $150 \mu \mathrm{g}$ twice daily or best tolerated dose is probably still the best option. The addition of a three times a day dosage schedule for the smallest dose $(50 \mu \mathrm{g})$ might improve the response rate without significantly decreasing tolerance.

In conclusion, iloprost caused a greater reduction in the severity of the attacks and the patients' opinion was also in favour of iloprost over placebo. The effects of iloprost on the total duration of Raynaud's attacks were greater than placebo, but did not reach statistical significance; the high placebo response rate and variability of data probably contributed to this. Furthermore, although this was one of the largest therapeutic studies of RS in SSc, the relatively small numbers may also have led to a type 2 error. Future studies to investigate this compound further should include larger numbers of patients and a three times a day dosage of $50 \mu \mathrm{g}$.

The authors thank Dr Mike Thompson, formerly of Schering Chemicals Ltd, for his help in excuting this study and preparing this manuscript. We are also indebted to Schering Chemicals AG for the supply of iloprost and matching placebo.

1 Allen E V, Brown G E. Raynaud's disease: A critical review of minimal requisites for diagnosis. Am $\mathcal{F}$ Med Sci 1932; 183: $187-200$.

2 Belch J J F. The phenomenon syndrome and disease of Maurice Raynaud. Brf Rheumatol 1991; 29: 162-5.

3 Rodeheffer R J, Rommer J A, Wigley F, Smith C R. Controlled double-blind trial of nifedipine in the treatment of Raynaud's phenomenon. $N$ Engl f Med 1983; 308: $880-3$.

4 Rademaker M, Cooke E D, Almond N E, et al. Comparison of intravenous infusions of iloprost and oral nifedipine in treatment of Raynaud's phenomenon in patients with systemic sclerosis: a double blind randomised study. $B M \mathcal{f}$ 1989; 298: 561-4.

5 Belch J J F, Newman P, Drury J K, et al. Intermittent epoprostenol (prostacyclin) infusion in patients with Raynaud's syndrome. Lancet 1981; i: 313-5.

6 Dowd P M, Martin M F R, Cooke E D, et al. Therapy of Raynaud's phenomenon by intravenous infusion of prostacyclin $\left(\mathrm{PGI}_{2}\right)$. Br $\mathcal{F}$ Dermatol 1982; 81: 106-10.

7 Rademaker M, Thomas R H M, Provost G, Beacham J A Cooke E D, Kirby J D. Prolonged increase in digital blood flow following iloprost infusion in patients with systemic flow following iloprost infusion in patients

8 Torley H I, Madhok R, Capell H A, et al. A double blind, randomised, multi center comparison of 2 doses of intravenous iloprost in the treatment of Raynaud's phenomenon secondary to connective tissue diseases. $A n$ Rheum Dis 1991; 50: 800-4.

9 Seibold J R, Wigley F M. Placebo controlled pilot study of intravenous iloprost in patients with Raynaud's phenomenon secondary to systemic sclerosis. F Rheumatol 1992; 19: 1407-14.

10 Lewis T. Raynaud's disease and pre-ganglionic sympathectomy. Clin Sci 1938; 3: 320-36.

11 Belch J J F, O'Dowd A, Forbes C D, Sturrock R D. Platelet sensitivity to a prostacyclin analogue in systemic sclerosis. Br ₹ Rheumatol 1987; 24: 346-50.

12 Dowd P M, Kirby J D, Holborow E J, Cooke E D Bowcock S A. Detection of immune complexes in systemic sclerosis and Raynaud's phenomenon. $B r f$ Dystemic sclerosis and Raynat 1981 ; 105: 179-88.

13 Belch J J F, Saniabadi A, Forbes C D. The effect of ZK 36374 (Iloprost) on white cell behaviour. In: Schror K, Gryglewski R J, eds. Prostacyclin and its stable analogue iloprost. Berlin: Springer Verlag, 1986; 97-102.

14 Belch J J F, Saniabadi A, Dickson R, Sturrock R D Forbes C D. Effect of Iloprost (ZK 36374) on white cel behaviour. In: Gryglewski R J, Stock G, eds. Prostacyclin and its stable analogue iloprost. Berlin: Springer Verlag, 1987; 97-102.

15 Dowd P M, Kovacs I B, Bland C J H, Kirby J D T. Effect of prostaglandins $I_{2}$ and $E_{2}$ on red cell deformability in patients with Raynaud's phenomenon and systemic patients with Raynaud's phenc.

16 Utsunomiya T, Kransz M M, Valeri C R, Shepro D, Hechtman H B. Treatment of pulmonary embolism with Hechtman H B. Treatment of pulmon

17 Belch J J F, Greer I, McLaren M, et al. The effrects of intravenous ZK36-374, a stable prostacyclin analogue, on intravenous ZK36-374, a stable prostacyclin analogu 